upregulated upon TDP-43 depletion. The downregulation of most of these transcripts largely correlates with binding of TDP-43 to large introns. TDP-43 also binds to the 3' UTR of some mRNAs, namely the FUS and neurofilament light chain-3 mRNAs, and furthermore the mRNAs for HDAC6 and for casein kinases 1 and 2, which can phosphorylate TDP-43 protein. TDP-43 also binds to its own pre-mRNA. The TDP-43 mRNA contains a long UG-rich region in its $3^{\prime}$ UTR where TDP-43 binds and thus appears to negatively regulate its own mRNA and protein levels.

These studies also bring up another point. TDP-43 binds to UG-rich regions of many RNA targets, but it cannot be predicted from the presence of UG sequences whether TDP-43 binds or not. This suggests that other determinants of specificity contributepossibly other proteins that associate with TDP-43 to recognize specific targets ${ }^{9}$, including the survival motor neuron (SMN) protein $^{13}$, which is deficient in spinal muscular atrophy. Mutations associated with TDP-43 in ALS and FTLD are enriched mainly in the glycine-rich C-terminal domain where most of these proteins bind, rather than in the regions encoding the RNA-recognition motifs. Thus, it is also possible that alterations in the interaction with other proteins make a major contribution to the pathomechanism of neurodegeneration in ALS and FTLD. Moreover, there remains the distinct possibility that alterations in RNA expression, stability and splicing are only a consequence rather than basis of a pathogenic mechanism involving TDP-43 in ALS and FTLD, at least in those cases caused by mutations in the Tdp43 gene.

Even if we were to set aside these reservations, this multitude of TDP-43 targets makes it difficult to decide which of them are most important for disease pathology. Moreover, neither study helps settle a major question in ALS and FTLD pathology: is disease pathology caused by a loss of nuclear function or gain of cytoplasmic function of the TDP-43 protein? Despite this, they may provide some clues for deciphering neuronal TDP-43 function in future studies. Genetic studies aimed at elucidating the function of TDP-43 have not been very helpful, mainly because TDP-43 knockout is lethal for mice from embryonic day 3.5 (refs. 14,15), long before motor neurons or cortical neurons differentiate. These new studies 7,8 therefore provide new testable hypotheses. We can now ask whether TDP-43 autoregulation leads to enhanced TDP-43 protein production upon nuclear depletion of TDP-43, resulting in enhanced export from the nucleus and cleavage of the cytoplasmic TDP-43, and whether this mechanism then supports aggregate formation that could promote disease progression in ALS and FTLD. We can also investigate whether altered regulation of noncoding RNAs or altered stability of mRNAs such as neurexin-3 and/or SLC1A2 after nuclear TDP-43 depletion is relevant for disease. Understanding the exact mechanism that leads to ALS or FTLD pathology may still be a long time coming, but these two studies help us take a step in the right direction.

\section{COMPETING FINANCIAL INTERESTS}

The author declares no competing financial interests.

1. Neumann, M. et al. Science 314, 130-133 (2006).

2. Mackenzie, I.R.A., Rademakers, R. \& Neumann, M. Lancet Neurol. 9, 995-1007 (2010).

3. Krecic, A.M. \& Swanson, M.S. Curr. Opin. Cell Biol. 11, 363-371 (1999).

4. Buratti, E. \& Baralle, F.E. Front. Biosci. 13, 867-878 (2008).

5. Ayala, Y.M. et al. J. Cell Sci. 121, 3778-3785 (2008).

6. Buratti, E. \& Baralle, F.E. J. Biol. Chem. 276, 36337-36343 (2001)

7. Tollervey, J.R. et al. Nat. Neurosci. 14, 452-458 (2011).

8. Polymenidou, M. et al. Nat. Neurosci. 14, 459-468 (2011).

9. Sephton, C.F. et al. J. Biol. Chem. 286, 1204-1215 (2011).

10. König, J. et al. Nat. Struct. Mol. Biol. 17, 909-915 (2010).

11. Reed, R. \& Magni, K. Nat. Cell Biol. 3, E201-E204 (2001).

12. Buratti, E. et al. EMBO J. 20, 1774-1784 (2001).

13. Wang, I.F., Reddy, N.M. \& Shen, C.K. Proc. Natl. Acad. Sci. USA 99, 13583-13588 (2002).

14. Sephton, C.F. et al. J. Biol. Chem. 285, 6826-6834 (2010).

15. Wu, L.S. et al. Genesis 48, 56-62 (2010).

\title{
CLC-3 spices up GABAergic synaptic vesicles
}

\section{Gudrun Ahnert-Hilger \& Reinhard Jahn

\begin{abstract}
How does chloride enter synaptic vesicle, and is it required for neurotransmitter uptake? A new study finds that the chloride transporter CLC-3 is needed for both acidification and transmitter loading of GABAergic synaptic vesicles.
\end{abstract}

Synaptic vesicles are the storage organelles for neurotransmitters. They are filled by specific transporters that operate as proton exchangers, drawing on an electrochemical proton gradient across the vesicle membrane. Vesicular neurotransmitter transporters control which transmitter is pumped into a synaptic vesicle and thus define which neurotransmitter is released from a given neuron.

Gudrun Ahnert-Hilger is at AG Functional Cell Biology, Institute for Integrative Neuroanatomy, Charité Universitätsmedizin Berlin, Berlin, Germany. Reinhard Jahn is in the Department of Neurobiology, Max Planck Institute for Biophysical Chemistry, Göttingen, Germany.

e-mail: rjahn@gwdg.de
The energy for transport is provided by a proton ATPase of the vacuolar class (V-ATPase). As in other acidic organelles, the ATPase is electrogenic and builds up a membrane potential, whereas effective proton pumping and the generation of a $\mathrm{pH}$ gradient require the influx of charge-balancing anions ${ }^{1,2}$. Chloride ions provide the charge balance during proton transport. Indeed, chloride ions are needed for acidification of synaptic vesicles. However, it has been surprisingly difficult to identify the chloride channel or transporter responsible.

In this issue of Nature Neuroscience, Riazanski et al. ${ }^{3}$ take a closer look at the role of CLC-3 in the function of GABAergic synaptic vesicles. CLC-3 is a member of the chloride channel/ chloride transporter (CLC) family, proteins that have long been prime candidates for providing charge balance in acidic organelles ${ }^{4,5}$. CLC-3 is widely expressed inside and outside of the brain and predominantly resides on intracellular trafficking vesicles, including synaptic vesicles ${ }^{4}$. During membrane recycling, pools of CLC-3 appear transiently at the plasma membrane ${ }^{6}$ and endocytosis may be facilitated by a direct interaction with clathrin ${ }^{7}$. As with other CLC family members, CLC- 3 probably acts as a $\mathrm{H}^{+} / \mathrm{Cl}^{-}$antiporter ${ }^{5}$. So far, only one direction of chloride transport, either into synaptic vesicles or outwardly rectifying at the plasma membrane, has been observed ${ }^{5,8}$.

If CLC-3 were the dominant pathway for chloride entry into synaptic vesicles, CLC-3 would be essential for generating a 

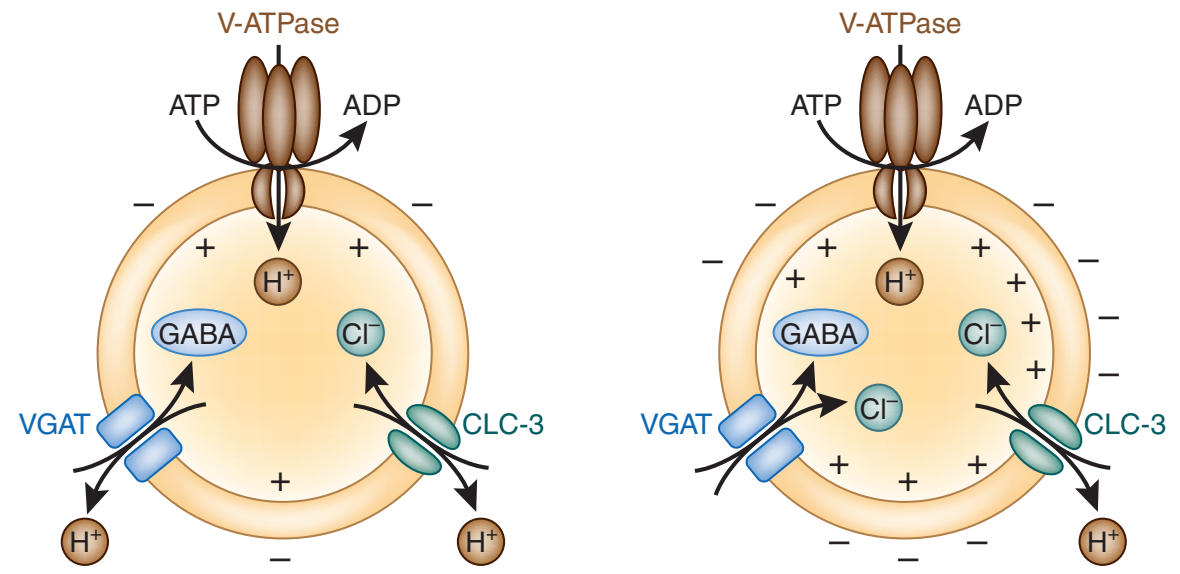

Figure 1 Two different models explaining the mechanism of GABA loading by synaptic vesicles. Left, VGAT may operate as a GABA-proton exchanger. Here, the V-ATPase establishes a pH gradient, with chloride ions that are imported by CLC-3 serving as charge balance during proton pumping. According to this model, VGAT operates as an electrogenic proton-GABA exchanger. Transport is driven by both the $\mathrm{pH}$ gradient (proton exchange) and the inside positive membrane potential. Right, VGAT may operate as a GABA-chloride co-transporter. Protons are not involved in the transport cycle. Transport is exclusively driven by the membrane potential, but, owing to the co-transport of chloride, $\mathrm{HCl}$ accumulates, particularly if CLC-3 transports chloride inward (as indicated in the figure). Alternatively, CLC-3 may provide compensation by shunting chloride out, with the protons leaking out again through the $\mathrm{V}_{0}$ proton channel, thus helping to disperse the $\mathrm{HCl}$ that accumulates during GABA transport.

$\mathrm{pH}$ gradient and for loading of vesicles with transmitter, at least for those transmitters depending primarily on a $\mathrm{pH}$ gradient, such as monoamines, acetylcholine and GABA. Surprisingly, however, this is not the case. Although deletion of CLC-3 in mice results in severe postnatal degeneration of the retina and the hippocampus, vesicle acidification is reduced but not abolished. Furthermore, no gross change in the amplitude of miniature postsynaptic currents, which scale with the vesicular transmitter content, is observed in neurons obtained from mice before the onset of degeneration ${ }^{4}$. Thus, the function in vesicular neurotransmitter uptake of CLC-3, so far the only CLC that has been unequivocally identified in synaptic vesicle populations, remains obscure.

Using hippocampal slices obtained from CLC-3 knockout mice before the onset of degeneration, Riazanski and colleagues ${ }^{3}$ found that the amplitude of GABAergic miniature inhibitory postsynaptic currents (mIPSCs) was reduced in the knockout slices. This finding indicates that there is a defect in transmitter release that was not found when the CLC-3 knockout mice were analyzed at an earlier age ${ }^{4}$. Lack of CLC-3 may lead to a diminished $\mathrm{pH}$ gradient across the vesicle membrane, resulting in inefficient vesicle loading (Fig. 1). To confirm that the diminished $\mathrm{pH}$ gradient is indeed the prime culprit, the authors incubated the slices with the basic buffer Tris, which enters synaptic vesicles during vesicle recycling and retards acidification by buffering away the protons. As predicted, both mIPSCs and evoked IPSCs were reduced. Furthermore, the reduction was more profound in slices derived from CLC-3-deficient mice, confirming that CLC-3 is required for the generation or maintenance of a pH gradient in GABAergic synaptic vesicles.

To directly measure whether vesicular proton pumping depends on CLC-3 as an entry pathway for charge-balancing chloride ions, the authors isolated synaptic vesicles from wild-type and knockout brains and measured ATP-dependent acidification. No substantial difference was observed (consistent with ref. 9) except for a shift in acidification kinetics. However, synaptic vesicle fractions are dominated by glutamatergic vesicles and it has been shown (albeit contested recently, see below) that the vesicular glutamate transporter may also serve as a chloride transporter ${ }^{9}$. The authors then used an elegant combination of immuno-isolation and immuno-depletion experiments to remove glutamatergic vesicles and to enrich GABAergic vesicles. In this VGLUT1-depleted fraction, vesicular acidification was substantially reduced in vesicles derived from CLC-3 knockout brains, directly confirming the theory that CLC-3 provides chloride ions as charge balance during proton pumping by the V-ATPase.

Riazanski et $a l^{3}$ document the need for CLC-3 in the filling of synaptic vesicles with GABA. Their results have implications for the transport mechanism of the vesicular
GABA transporter VGAT (also referred to as VIAAT). Previous uptake studies using intact or reconstituted vesicles found that vesicular GABA uptake primarily depends on a $\mathrm{pH}$ gradient, although a contribution of the membrane potential was also observed (as expected if the transport cycle is electrogenic) $)^{1,2}$. The results of Riazanski et al. ${ }^{3}$ are consistent with this view and indicate that $\mathrm{pH}$ gradients are essential for GABA uptake. Thus, these data suggest that VGAT operates as a proton-GABA exchanger (Fig. 1). According to this scenario, chloride influx maintains the $\mathrm{pH}$ gradient during the transport cycle. This cycle starts with protons being pumped into the vesicle by the V-ATPase, which are in turn shifted out in exchange for GABA. This electroneutral 'conveyor belt' model nicely explains how a synaptic vesicle can be filled with thousands of molecules of GABA, even though only a few protons (even including those absorbed by buffering) are needed to create a full-scale $\mathrm{pH}$ gradient under steady-state conditions. The ion balance for sustaining the $\mathrm{pH}$ gradient is maintained by chloride entering vesicles through CLC- 3 and perhaps by VGLUTs ${ }^{9}$ in subpopulations of GABAergic vesicles ${ }^{10}$.

This view, however, is difficult to reconcile with a recent study ${ }^{11}$ in which purified VGAT was reconstituted in proteoliposomes and GABA transport was studied in the presence of either a co-reconstituted proton ATPase or artificially imposed diffusion potentials. The data from that study suggest that VGAT operates as a chloride co-transporter that is driven by an inside positive membrane potential. Furthermore, the authors excluded the possibility that VGAT functions as a GABAproton exchanger in their reconstituted system (Fig. 1). Vesicle filling with GABA is more difficult to explain by this mechanism. Because there is no indication that CLC-3 operates in the reverse direction ${ }^{5}$ and because CLC-3 exchanges chloride for protons, the previously suggested transport mechanism ${ }^{11}$ would lead to an accumulation of massive amounts of hydrochloric acid inside the vesicle during GABA loading. Thus, despite the exciting new findings, there is still much to do before we understand how synaptic vesicles are loaded with thousands of GABA molecules in less than a minute, particularly when considering that charge neutrality must be maintained at each transport cycle and that the high amounts of $\mathrm{NaCl}$ picked up during endocytosis must be displaced to maintain osmotic balance during transport.

The data of Riazanski et al. ${ }^{3}$ may explain, at least to a certain extent, the molecular origin of the severe pathologies of CLC-3 
knockout mice. The massive degeneration of the hippocampus and retina ${ }^{4}$ may be caused by a dysfunction of GABAergic interneurons. However, cerebellar function is only mildly if at all impaired in CLC-3 knockouts, despite the high CLC-3 expression in this area ${ }^{12}$. In addition, other areas in which GABAergic neurons are dominant, such as the striatum, do not show similar degeneration. It is conceivable that other chloride import pathways (involving, for instance, CLC-7 (ref. 5) or VGLUT2, with the latter being known to reside on GABAergic synaptic vesicles ${ }^{10}$ ) compensate for CLC-3 in these areas. However, the possibility cannot be excluded that degeneration is not related to GABAergic dysfunction but is instead primarily caused by a defect in the function of endosomes, where CLC-3 is known to mediate acidification ${ }^{5}$.

In conclusion, Riazanski et al. $^{3}$ find that CLC-3 is an important factor for proper filling of GABAergic vesicles and for GABAergic transmission. This contribution underscores the importance for synaptic plasticity of a tightly controlled ionic balance at the vesicular level.

\section{COMPETING FINANCIAL INTERESTS}

The authors declare no competing financial interests.

1. Ahnert-Hilger, G., Höltje, M., Pahner, I., Winter, S. \& Brunk, I. Rev. Physiol. Biochem. Pharmacol. 150, 140-160 (2003).
2. Edwards, R.H. Neuron 55, 835-858 (2007).

3. Riazanski, V. et al. Nat. Neurosci. 14, 487-494 (2011)

4. Stobrawa, S.M. et al. Neuron 29, 185-196 (2001)

5. Jentsch, T.J. J. Physiol. (Lond.) 578, 633-640 (2007).

6. Zhao, Z., Li, X., Hao, J., Winston, J.H. \& Weinman, S.A. J. Biol. Chem. 282, 29022-29031 (2007).

7. Stauber, T. \& Jentsch, T.J. J. Biol. Chem. 285 34537-34548 (2010).

8. Li, X., Shimada, K., Showalter, L.A. \& Weinman, S.A. J. Biol. Chem. 275, 35994-35998 (2000).

9. Schenck, S., Wojcik, S.M., Brose, N. \& Takamori, S Nat. Neurosci. 12, 156-162 (2009).

10. Zander, J.F. et al. J. Neurosci. 30, 7634-7645 (2010).

11. Juge, N. \& Muroyama, A. J. Biol. Chem. 284 35073-35078 (2009).

12. Dickerson, L.W. et al. Brain Res. 958, 227-250 (2002).

\section{The multi-laned hippocampus}

\section{Edvard I Moser}

\section{Few brain circuits have generated more research than the mammalian hippocampus, a region of discrete subfields that connect serially to form a 'trisynaptic loop'. A new paper implies that the loop may be made up of parallel subpathways.}

One of the first mammalian brain regions to attract the founders of modern neuroscience was the hippocampus ${ }^{1}$, a phylogenetically conserved three-layered cortex known today to contribute critically to storage of memory. Incoming signals from the entorhinal cortex are passed unidirectionally through the hippocampus through a 'trisynaptic loop' consisting of synapses between principal cells in three major subfields: the dentate gyrus, CA3 and CA1 (Fig. 1). Although each subfield also receives direct cortical input, the intrinsic circuit is often thought to enable a step-wise transformation of incoming information into a network code efficient for memory storage and retrieval ${ }^{2,3}$. However, although the recognition of subfield differences has greatly improved our understanding of hippocampal computation, the complexity of the circuit may still be vastly underestimated. Recent work has identified considerable variability in patterns of gene expression within each of the major CA subfields ${ }^{4,5}$, as well as differences in temporal firing patterns among subgroups of CA1 cells ${ }^{6}$. There is no doubt that the subfields are heterogeneous, but whether the various genetic and functional subpopulations form distinct neural circuits has not been determined.

Edvard I. Moser is at the Kavli Institute for Systems Neuroscience, Norwegian University of Science and Technology, Trondheim, Norway.

e-mail: edvard.moser@ntnu.no

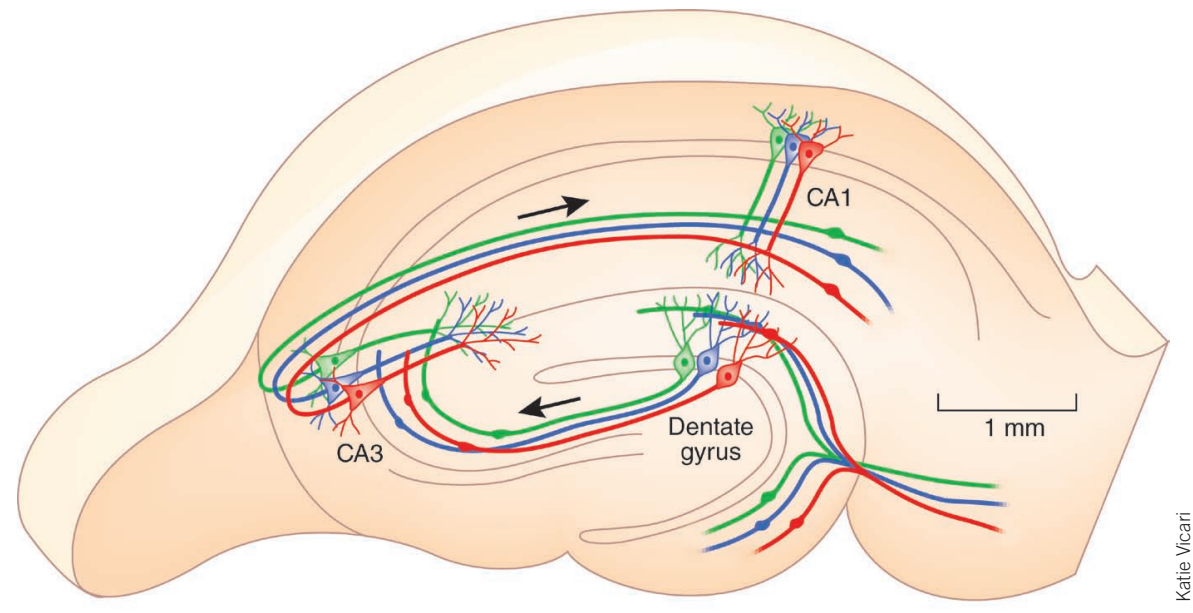

Figure 1 Parallel pathways through the hippocampus. Neurons with similar reporter genes in dentate gyrus, CA3 and CA1 form connections with each other. Connectivity with other neuron groups is more limited. The figure shows connections between mGFP-expressing neurons in Lsi1 and Lsi2 mice (red and blue, respectively). The possibility of additional connectivity channels (green) is indicated.

In this issue, Deguchi et al. ${ }^{7}$ provide evidence for selective connectivity between hippocampal neurons with a common molecular marker. They studied circuit formation postnatally in transgenic mouse lines expressing a fluorescent protein under the control of a Thy1.2 promoter cassette ${ }^{8}$. In several such Thyl lines, the reporter gene is expressed only in a small subpopulation of principal neurons, enabling researchers to study neuronal connections on a relatively silent background, as in a classical Golgi preparation $^{9}$. Deguchi and colleagues exploited the sparse expression of membrane-targeted green fluorescent protein (mGFP) in two of these lines, Lsi1 and Lsi2, to determine how genetically defined neuronal subpopulations connect in the developing hippocampus. mGFP-labeled cells emerged earlier in Lsi1 than Lsi2, and synapse formation was staggered by several days. Surprisingly, in each mouse line, labeled neurons connected extensively to other labeled neurons, but not as much to the rest of the cell population. In mice 1 to several months old, mossy fiber terminals on Lsil pyramidal dendrites in CA3 